\title{
Early diagnosis of tuberculous meningitis by an indirect ELISA protocol based on the detection of the antigen ESAT-6 in cerebrospinal fluid
}

\author{
F. Song $\cdot$ X. Sun $\cdot$ X. Wang $\cdot$ Y. Nai $\cdot$ \\ Z. Liu
}

Received: 20 July 2012/ Accepted: 16 June 2013/Published online: 18 July 2013

(c) The Author(s) 2013. This article is published with open access at Springerlink.com

\begin{abstract}
Background Early diagnosis of tuberculous meningitis (TBM) is still a challenge; the present study aimed to establish a method of detecting the antigen early secreted antigenic target 6 (ESAT-6) in cerebrospinal fluid (CSF) by an indirect enzyme-linked immunosorbance assay (ELISA) protocol and to study the value of detecting ESAT-6 in CSF in the early diagnosis of TBM.

Methods An indirect ELISA protocol was used, employing a monoclonal antibody (mAb) against ESAT-6, which was used to demonstrate ESAT-6 in the CSF from TBM patients and non-TBM controls. CSF was obtained from 100 patients: confirmed TBM, clinically diagnosed TBM, disease controls, and healthy controls $(n=10)$. Pure recombinant ESAT-6 (standard product) was used in serial dilutions to detect the absorbance and to establish a standard curve from the data; the concentration was on the $X$ axis vs. absorbance on the $Y$ axis, and the standard curve was used to interpolate the concentration of ESAT- 6 in samples.

Results The indirect ELISA method provided $88 \%$ sensitivity and $92 \%$ specificity for the diagnosis of TBM using a $\mathrm{mAb}$ to ESAT-6. The mean concentration of ESAT-6 in TBM patients was significantly higher than that of the non-TBM groups. There was also a significant difference in the mean ESAT-6 expression between the confirmed TBM patients and the clinically diagnosed TBM patients $(p<0.01)$.

Conclusions Detection of ESAT- 6 in the CSF of TBM patients by indirect ELISA protocol gives a reliable early diagnosis and can be used to develop an immunodiagnostic assay with increased sensitivity and specificity.
\end{abstract}

F. Song $\cdot$ X. Sun $(\bowtie) \cdot X$. Wang $\cdot$ Y. Nai $\cdot$ Z. Liu

Department of Neurology, Yantai Yuhuangding Hospital, Yantai 264000, Shandong Province, China

e-mail: fuxia99@yeah.net
Keywords Tuberculous meningitis - Tuberculosis . Cerebrospinal fluid · ESAT-6 · ELISA · Diagnosis

\section{Introduction}

Tuberculosis (TB) remains a major public health problem in the twenty-first century. A third of the world's population is infected with Mycobacterium tuberculosis (MTB); among them, 5-10\% of the infected population will develop the disease during their lifetime, and TBM affects about 7-12\% [1] of all tuberculosis patients. However, about $30 \%$ of patients with TBM will die despite antituberculosis chemotherapy [2]. TB is responsible for $>2$ million deaths per year worldwide. The situation is exacerbated by co-infection with human immunodeficiency virus (HIV).

The diagnosis of TBM relies on the isolation of MTB from CSF, but direct staining of CSF for acid-fast bacilli has very low sensitivity (10-20\%) [3]; bacilli cultures usually take 4-6 weeks and also lack sensitivity (25-70\%) $[4,5]$. Yet, timely diagnosis and treatment will have a direct influence on the prognosis. So, new, affordable, sensitive, specific diagnostic assays are required.

In the present study, we used the method of indirect ELISA to detect the mycobacterial antigen ESAT-6. ESAT-6 is an early secreted and low molecular weight protein of MTB, and exists mainly in pathogenic mycobacteria but not in all $M$. bovis BCG strains or in most environmental mycobacterials examined so far [6]. Previous studies on ESAT-6 concentrated mainly on specific T cell responses and gamma interferon (IFN- $\gamma$ ) induced by ESAT-6. To our knowledge, there are no reports on the quantitative detection of ESAT-6 in the CSF of TBM patients. 


\section{Methods}

Study subjects

We selected 100 patients from an inpatient department (73 males, 27 females, within the ages of 13-68 years); 10 had confirmed TBM (the direct staining of the CSF for acid-fast bacilli and/or cultures for bacilli were positive), 40 were diagnosed by clinical symptoms based on the criterion of Thwaites [5]. All cases above were diagnosed in $<2$ weeks after the onset of their disease, and the cases were more severe in the confirmed TBM group; four with active pulmonary tuberculous, four with tuberculoma, and two with spinal cord involvement. Non-TBM diseases $(n=50)$ were selected for the control, including multiple sclerosis $(n=10)$, acute myelitis $(n=10)$, viral meningitis $(n=10)$, purulent meningitis $(n=8)$, cryptococcal meningitis $(n=2)$, and healthy controls $(n=10)$. Samples were collected from all study groups for which patient's consent and the ethical committee's approval was obtained.

Antigen and antibody

The recombinant pure antigen ESAT-6 (standard product) was bought from ProSpec (USA). The primary mAb against ESAT-6 and the secondary mAb (peroxidase conjugate goat anti-mouse IgG) were bought from Abcam (UK).

\section{Specimens}

CSF was obtained from the 100 patients by puncture as soon as they were diagnosed and stored at $-80{ }^{\circ} \mathrm{C}$ until it use.

Establishing a standard curve

Prior to patient sampling, the assay was standardized by incubating purified ESAT-6 with the mAb against ESAT-6 at different dilutions, using the indirect ELISA method (see the method in following text) to detect the absorbance of the standard product at different dilutions and to establish a standard curve, with concentration on the $X$ axis vs. absorbance on the $Y$ axis (Fig. 1). The different dilutions of the standard product were prepared by diluted in coating buffer (3.03 $\mathrm{g} \mathrm{Na}_{2} \mathrm{CO}_{3}, 6.0 \mathrm{~g} \mathrm{NaHCO}_{3}, 1,000 \mathrm{ml}$ distilled water, $\mathrm{pH} 9.6)$ at $1,2,5,10,25,50,100$, and $200 \mathrm{ng} / \mathrm{ml}$.

\section{Indirect ELISA protocol}

CSF samples in a volume of $50 \mu \mathrm{l}$ (the same as the standard product) from TBM patients and control subjects were added to each well, and incubated for $2 \mathrm{~h}$ at room

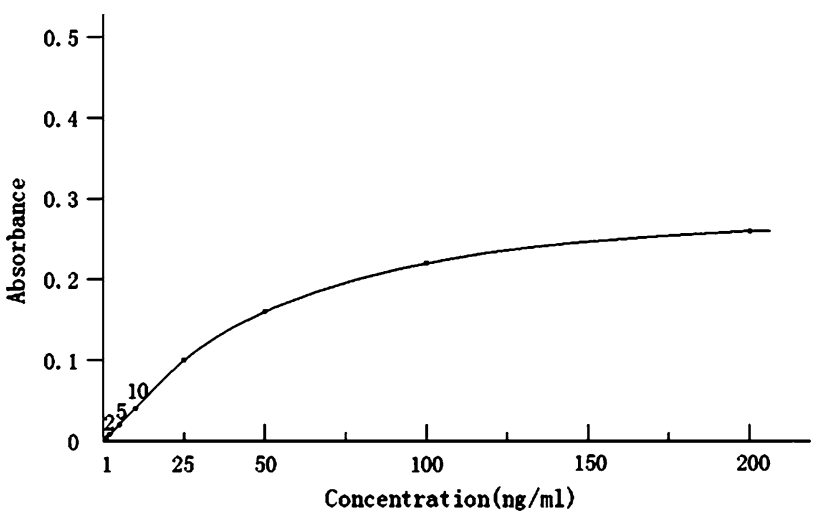

Fig. 1 The standard curve of ESAT- 6 antigen in CSF by the ELISA protocol developed in this study

temperature. Then, the plate was washed twice by filling the wells with $20 \mu \mathrm{l}$ of phosphate-buffered saline (PBS). The remaining protein-binding sites in the wells were blocked by adding $200 \mu \mathrm{l}$ of $5 \%$ non-fat dry milk per well, then incubated overnight at $4{ }^{\circ} \mathrm{C}$ and washed five times with $200 \mu$ of PBS. The primary mAb (1:3,200, diluted in $5 \%$ non-fat dry milk) was added $(100 \mu \mathrm{l})$ in each well and incubated for $2 \mathrm{~h}$ at room temperature in the dark. The wells were then washed five times with $200 \mu 1$ of PBS. The secondary antibody $(1: 5,000$, diluted in 5\% non-fat dry milk; $100 \mu \mathrm{l}$ ) was added to each well and incubated for $2 \mathrm{~h}$ at room temperature in the dark. Then, the plate was washed five times with $200 \mu \mathrm{l}$ of PBS; $100 \mu \mathrm{l}$ the SUBSTBM substrate was added to the wells and incubated at room temperature for $15 \mathrm{~min}$, also in the dark. The reaction was then stopped with $100 \mu \mathrm{l}$ of $0.3 \mathrm{M} \mathrm{H}_{2} \mathrm{SO}_{4}$ (stopping buffer) after sufficient color development. The absorbance of each well was read at $450 \mathrm{~nm}$. Then, we calculated the concentration of each sample from the standard curve.

Statistical analysis

Results are reported as mean \pm standard deviation; differences between the TBM patient groups and controls and between the confirmed TBM group and the clinically diagnosed group were analyzed by $t$ tests using SPSS software (version 13; SPSS Inc, Chicago, IL, USA). The adopted level of statistical significance was $p<0.05$.

\section{Results}

The minimum antigen level that could be detected was $1 \mathrm{ng} / \mathrm{ml}$, so patients with a level of ESAT- 6 in the CSF greater than $1 \mathrm{ng} / \mathrm{ml}$ were considered positive cases. The proportions of CSF cases positive for ESAT- 6 in the confirmed and clinically diagnosed TBM patients were $90 \%$ 
(9/10) and $87.5 \%(35 / 40)$, respectively, while the positive rate for patients in the control group was $8 \%(4 / 50)$. Overall, the indirect ELISA method provided $88 \%$ sensitivity and $92 \%$ specificity for the diagnosis of TBM using a mAb to ESAT-6. The mean concentration of ESAT-6 in the CSF of TBM patients was $85.13 \pm 18.18 \mathrm{ng} / \mathrm{ml}$, which was significantly higher than that of the non-TBM groups $(2.76 \pm 1.57 \mathrm{ng} / \mathrm{ml}, p<0.001)$. There was also a significant difference in the mean ESAT-6 expression between the confirmed TBM patients $(112.87 \pm 16.67 \mathrm{ng} / \mathrm{ml})$ and the clinically diagnosed TBM patients $(78.20 \pm 10.22 \mathrm{ng} /$ $\mathrm{ml}, p<0.001)$. In the TBM patient group, the level of ESAT- 6 in the CSF was $>70 \mathrm{ng} / \mathrm{ml}$ in 24 cases, and 14 of them had neurological sequelae, such as headache, epilepsy, or limb weakness. Patients with an ESAT-6 level below $70 \mathrm{ng} / \mathrm{ml}$ had no neurological sequelae (Table 1).

\section{Discussion}

Early diagnosis of TBM is still difficult; besides conventional experimental methods, immunoassays have been recently adopted. Various immunoassays for detecting antigens or antibodies in the serum or CSF of TBM patients have been developed with different sensitivities and specificities. A purified protein derivative (PPD) from MTB, i.e. a complex mixture of antigens, has long been used as a skin test for TB diagnosis. The tuberculin skin test (TST) is technically simple, but it lacks diagnostic specificity in BCG-vaccinated individuals. Another widely used diagnostic test is polymerase chain reaction (PCR) using specific primers as markers for MTB, but this test is not performed correctly in all clinical laboratories and shows variable sensitivity and specificity. Many serological assays have been tried, but nearly all have failed to improve upon the time-honored sputum smear and culture approach. Currently, it is believed that antigen detection is more sensitive than antibody detection [7], especially specific MTB antigens such as ESAT-6 and the Ag85 complex. However, the early phase of MTB infection, in patients treated with corticosteroids, or in immune-deficient TBM patients, these tests may provide false negative results when antibody detection is used, and antibody detection cannot distinguish whether it is an acute infection

Table 1 The mean concentration of ESAT-6 in CSF in each group

\begin{tabular}{lcc}
\hline Group & $n$ & Concentration $(\mathrm{ng} / \mathrm{ml})$ \\
\hline Confirmed TBM patients & 10 & $112.87 \pm 16.67$ \\
Clinically diagnosed TBM patients & 40 & $78.20 \pm 10.22$ \\
Control & 50 & $2.76 \pm 1.57$ \\
\hline
\end{tabular}

or a latent infection. Therefore, there has been growing interest in antigen detection to diagnose TBM [8].

Since the identification of MTB ESAT-6, there has been growing interest in the use of this antigen to diagnose TB. In previous studies, it was demonstrated that most TB patients (35-92\%) can recognize ESAT-6, while healthy unrelated controls do not [8-12]. ESAT-6 is an immunodominant $\mathrm{T}$ cell stimulatory antigen and is recognized by specific IFN- $\gamma$-secreting $\mathrm{T}$ cells present in greater numbers in patients who have an active infection compared with those who are uninfected with MTB [13]. Moreover, the numbers of specific IFN- $\gamma$-secreting $\mathrm{T}$ cells are significantly higher in the CSF than in the peripheral blood [14, 15]. ESAT-6-induced specific IFN- $\gamma$ responses have been shown to be useful in discriminating infected individuals from healthy controls [16-18]. Consequently, the possible use of ESAT-6 as a marker of Mycobacterium tuberculosis infection has been proposed in regions with low TB endemicity [19].

Murakami [15] reported a case that was diagnosed with TBM based on the detection of ESAT-6-specific IFN- $\gamma$ production in CSF by ELISPOT (an enzyme-linked immunospot assay). Mathai [20] reported that detecting PPD antigen in CSF by Dot-Iba (a dot-immunobinding assay) provided a sensitivity of $83.3 \%$ and specificity of $95 \%$. Similarly, Kashyap [21] also detected ESAT-6 by an indirect ELISA protocol; the sensitivity was $80 \%$ and the specificity was $86 \%$. Overall, these findings support our results, suggesting that the detection of ESAT- 6 in CSF might be developed into a diagnostic test for TBM.

In the present study, using an indirect ELISA method, we conducted a prospective study to demonstrate ESAT-6 in the CSF of TBM patients using a mAb against the antigen. The data demonstrate that positivity for ESAT-6 in cases of confirmed and clinically diagnosed TBM patients was $90 \%(9 / 10)$ and $87.5 \%$ (35/40), respectively, while the positivity for patients in the non-TBM group was $8 \%$ (4/50), the sensitivity of detecting ESAT-6 in CSF for diagnosing TBM was $88 \%$ and the specificity was $92 \%$. The minimum antigen level that could be detected was $1 \mathrm{ng} / \mathrm{ml}$, suggesting that the indirect ELISA method is quite sensitive. However, in the non-TBM group, four cases were positive. The reason for these false-positive results is not clear, as test error cannot be ruled out, but these levels of ESAT-6 were much lower than those in TBM patients $(p<0.001)$. Another finding is that, in the confirmed TBM group, the level of ESAT- 6 was higher than in the clinically diagnosed group $(p<0.001)$. This could be that confirmed, diagnosed TBM patients showed more severe clinical manifestations; so it can be speculated that the level of ESAT-6 in CSF is higher in more severe patients. In the TBM patient group, 14 cases with a level of ESAT-6 in the CSF $>70 \mathrm{ng} / \mathrm{ml}$ had neurological sequelae, 
but patients with a level of ESAT- $6<70 \mathrm{ng} / \mathrm{ml}$ had no neurological sequelae. Accordingly, the level of ESAT-6 in CSF can differentiate between severe infection and mild infection in TMB and could help clinicians estimate the prognosis of the disease. On the other hand, all the TBM cases in the present study were diagnosed $<2$ weeks after the onset of disease, and the indirect ELISA test can be completed in $24 \mathrm{~h}$. Therefore, the present assay is appropriate for early diagnosis of TBM with high sensitivity and specificity.

In this study, detection of ESAT-6 in TBM patients by indirect ELISA gave a reliable early diagnosis of TBM, and the test is rapid, sensitive, cost-effective, and could be performed in any standard pathology laboratory. It could be used for developing an immunodiagnostic assay with increased sensitivity and specificity.

\section{Conflict of interest None.}

Open Access This article is distributed under the terms of the Creative Commons Attribution License which permits any use, distribution, and reproduction in any medium, provided the original author(s) and the source are credited.

\section{References}

1. Hooker JA, Muhindi DW, Amayo EO, Mc'ligeyo SO, Bhatt KM, Odhiambo JA (2003) Diagnosis utility of cerebrospinal fluid studies in patients with clinically suspected tuberculous meningitis. Int J Tuberc Lung Dis 7:787-796

2. Thwaites G, Chau TT, Mai NT, Drobniewski F, McAdam K, Farrar J (2000) Tuberculous meningitis. J Neurol Neurosurg Psychiatry 68:289-299

3. Pai M, Flores LL, Pai N, Hubbard A, Riley LW, Colford JM Jr (2003) Diagnostic accuracy of nucleic acid amplification tests for tuberculous meningitis: a systematic review and meta-analysis. Lancet Infect Dis 3:633-643

4. Thwaites GE, Chau TT, Farrar JJ (2004) Improving the bacteriological diagnosis of tuberculous meningitis. J Clin Microbiol 42:378-379

5. Thwaites GE, Chau TT, Stepniewska K et al (2002) Diagnosis of adult tuberculous meningitis by use of clinical and laboratory features. Lancet 360:1287-1292

6. Ewer K, Deeks J, Alvarez L et al (2003) Comparison of T-cellbased assay with tuberculin skin test for diagnosis of Mycobacterium tuberculosis infection in a school tuberculosis outbreak. Lancet 361:1168-1173

7. Kashyap RS, Kainthla RP, Biswas SK et al (2003) Rapid diagnosis of tuberculous meningitis using the simple Dot ELISA method. Med Sci Monit 9:MT123-MT126
8. Arend SM, Andersen P, van Meijgaarden KE et al (2000) Detection of active tuberculosis infection by $\mathrm{T}$ cell responses to early-secreted antigenic target $6-\mathrm{kDa}$ protein and culture filtrate protein 10. J Infect Dis 181:1850-1854

9. Mustafa AS, Oftung F, Amoudy HA et al (2000) Multiple epitopes from the Mycobacterium tuberculosis ESAT-6 are recognized by antigen-specific human $\mathrm{T}$ cell lines. Clin Infect Dis 30:S201-S205

10. Ravn P, Demissie A, Eguale T et al (1999) Human T cell responses to the ESAT-6 antigen from Mycobacterium tuberculosis. J Infect Dis 179:637-645

11. Smith SM, Klein MR, Malin AS et al (2000) Human CD8+ T cells specific for Mycobacterium tuberculosis secreted antigens in tuberculosis patients and healthy BCG-vaccinated controls in The Gambia. Infect Immun 68:7144-7148

12. Ulrichs T, Munk ME, Mollenkopf H et al (1998) Differential T cell responses to Mycobacterium tuberculosis ESAT-6 in tuberculosis patients and healthy donors. Eur J Immunol 28:39493958

13. Hasan Z, Jamil B, Ashraf M et al (2009) ESAT6-Induced IFN- $\gamma$ and CXCL9 can differentiate severity of tuberculosis. PLoS ONE 4:e5158

14. Thomas MM, Hinks TS, Raghuraman S et al (2008) Rapid diagnosis of $M$. tuberculosis meningitis by enumeration of cerebrospinal fluid antigen-specific $\mathrm{T}$ cells. Int $\mathrm{J}$ Tuberc Lung Dis 12:651-657

15. Murakami S, Takeno M, Oka H et al (2008) Diagnosis of tuberculous meningitis due to detection of ESAT-6-specific gamma interferon production in cerebrospinal fluid enzymelinked immunospot assay. Clin Vaccine Immunol 15:897-899

16. Abramo C, Meijgaarden KE, Garcia D et al (2006) Monokine induced by interferon gamma and IFN-gamma response to a fusion protein of Mycobacterium tuberculosis ESAT-6 and CFP10 in Brazilian tuberculosis patients. Microbes Infect 8:45-51

17. Brock I, Munck ME, Kok-Jensen A, Anderson P (2001) Performance of whole blood IFN- $\gamma$ test for tuberculosis diagnosis based on PPD or the specific antigens ESAT- 6 and CFP-10. Int J Tuberc Lung Dis 5:462-467

18. Ulrichs T, Anding R, Kaufmann SH, Munk ME (2000) Numbers of IFN- $\gamma$ producing cells against ESAT- 6 increase in tuberculosis patients during chemotherapy. Int $J$ Tuberc Lung Dis 4:1181-1183

19. Cardoso FL, Antas PR, Milagres AS et al (2002) T-cell responses to the Mycobacterium tuberculosis-specific antigen ESAT-6 in Brazilian tuberculosis patients. Infect Immun 70:6707-6714

20. Mathai A, Radhakrishnan W, Sarada C, George SM (2003) Detection of heat stable mycobacterial antigen in cerebro-spinal fluid by Dot-Immunobinding assay. Neurol India 51:52-54

21. Kashyap RS, Ramteke SS, Morey SH, Purohit HJ, Taori GM, Daginawala HF (2009) Diagnostic value of early secreted antigenic target-6 for the diagnosis of tuberculous meningitis patients. Infection 37(6):508-513 